Claremont Colleges

Scholarship@ Claremont

All HMC Faculty Publications and Research

HMC Faculty Scholarship

$1-1-2010$

\title{
Stability and Dynamics of Self-Similarity in Evolution Equations
}

Andrew J. Bernoff

Harvey Mudd College

Thomas P. Witelski

Duke University

\section{Recommended Citation}

A. J. Bernoff and T. P. Witelski, Stability and dynamics of self-similarity in evolution equations, J. Eng. Math., 66 (2010) 11-31. doi: $10.1007 / \mathrm{s} 10665-009-9309-8$

This Article is brought to you for free and open access by the HMC Faculty Scholarship at Scholarship @ Claremont. It has been accepted for inclusion in All HMC Faculty Publications and Research by an authorized administrator of Scholarship @ Claremont. For more information, please contact scholarship@cuc.claremont.edu. 


\title{
Stability and Dynamics of Self-Similarity in Evolution Equations
}

\author{
Andrew J. Bernoff (ajb@hmc.edu)* \\ Department of Mathematics, Harvey Mudd College \\ Thomas P. Witelski (witelski@math.duke.edu) $)^{\dagger}$ \\ Department of Mathematics, Duke University
}

\begin{abstract}
We discuss a methodology for studying the linear stability of self-similar solutions. We will illustrate these fundamental ideas on three prototype problems: a simple ODE with finite-time blowup, a second-order semi-linear heat equation with infinite-time spreading solutions, and the fourth-order Sivashinsky equation with finite-time self-similar blow-up. These examples are used to show that self-similar dynamics can be studied using many of the ideas arising in the study of dynamical systems. In particular, we will discuss the use of dimensional analysis to derive scaling invariant similarity variables, and the role of symmetries in the context of stability of self-similar dynamics. The spectrum of the linear stability problem determines the rate at which the solution will approach a self-similar profile. For blow-up solutions we will demonstrate that the symmetries give rise to positive eigenvalues associated with the symmetries, and show how this stability analysis can identify a unique stable (and observable) attracting solution from a countable infinity of similarity solutions.
\end{abstract}

Keywords: Self-similarity, Scale-invariance, Stability, Dynamical Systems

\section{Introduction}

Self-similarity in an evolving system describes dynamics where the current solution can be rescaled (in time and/or space) to exactly reproduce later states of the system. Self-similar behavior arises naturally in a wide spectrum of applied settings and physical phenomena, and is captured in the mathematical evolution equations used to model these phenomena $[3,4,49,31]$.

For example, such self-similar behavior can be seen in many physical problems exhibiting diffusion, where a localized initial distribution of heat, mass, or energy will spread, and after a rescaling, approach a universal profile at large times. In the viscous spreading of a liquid drop on a flat surface, at large times the drop approaches an axisymmetric form with a unique spatial profile upon rescaling the radius and the height $[68,60]$. Other examples are the diffusion of a localized source of heat $[74,78,51,52]$ and the viscous spreading of vorticity in a two-dimensional fluid where the solutions approach a spreading Gaussian profile $[12,42]$.

In contrast to problems where the self-similar behavior continues indefinitely (called 'infinite-time' behavior), some systems form singularities in finite-time, usually associated with a change of topology or a physical quantity diverging. If the magnitude of the solution

\footnotetext{
* This research was supported in part by NSF Grant DMS-9971969, DMS-0807347 and internal grants from Harvey Mudd College.

$\dagger$ This research was supported by NSF Grant DMS-0239125 CAREER.
}

(C) 2011 Kluwer Academic Publishers. Printed in the Netherlands. 
is diverging, $|u| \rightarrow \infty$, the behavior is generally called finite-time blow-up. Blow-up has been studied extensively in reaction-diffusion equations in combustion theory [6] and mathematical biology [66]. Problems having the time-derivative of the solution diverging, $\left|u_{t}\right| \rightarrow \infty$, while the solution remains bounded are said to exhibit quenching [54]. In quenching, spatial gradients of the solution will diverge while the solution approaches a limit value, often $|u| \rightarrow 0$, and in different physical contexts similar behavior (with or without formation of a singularity in $u_{t}$ ) is called extinction, pinching, rupture, touch-down, or pull-in [40, 47, 37]. In the pinch-off of a thin liquid filament, as in a lava lamp or the break-up of a fluid jet, the profile of the pinching neck assumes a universal shape as detachment is approached [18, 32]. Other examples include rupture of a solid filament due to surface diffusion [11], rupture of thin films $[79,69,70,75,76]$, and the ignition of a combustible substance [6].

In this paper we review some of the tools that have developed in the past few decades for studying the dynamics associated with self-similar behavior. In seminal work, Barenblatt made use of dimensional analysis to classify the types of self-similar solutions observed as intermediate asymptotic states [3, 4]. This led to an explosion of work deriving, classifying, computing and rigorously analyzing self-similar solutions in a wide array of systems (see the reviews $[3,55,63,2,39,4,40,49,31]$ and references therein).

We concentrate on describing a linear stability theory for self-similar evolutions. To study stability the governing equation in original variables is transformed into an equation in similarity variables. The proper choice of similarity variables maps the similarity solution of the original problem to a steady state of the transformed equation. The dynamics are then linearized about this steady state. For PDE's, appropriate boundary conditions must be posed to complete the linear problem. For infinite-time spreading dynamics, it may be appropriate to assume that the solution is advancing into an undisturbed medium [74]. For finite-time singular behavior, boundary conditions are posed in a matching region that is near to the blow-up in primitive variables but lies in the far-field with respect to the self-similar variables $[75,76]$.

In systems where self-similar dynamics are approached, stability theory determines the rate at which solutions converge to a self-similar profile. Due to the symmetries of the original problem, self-similar dynamics always exist in continuous families of solutions with degrees of freedom tied to symmetry modes (e.g. translation invariance in time or space). This gives rise to the idea of optimal asymptotics [51, 52], where an optimal member of the parametrized family of similarity solutions is determined to maximize the rate of convergence to the solution from given initial conditions. Symmetry modes can be associated with the exponential separation of solutions on the manifold of similarity solutions generated by the physical symmetries (e.g. spatial and temporal translation, rotation, etc.) of the problem. Properly accounting for the linearized contributions of the symmetry modes selects the optimal self-similar solution. For finite-time blow-up, if the remainder of the linearized spectrum of a similarity solution is stable, non-optimal choices of symmetry parameters can be misinterpreted as instabilities of otherwise stable self-similar dynamics.

We use three examples to illustrate ideas: 
1. First, a third-order nonlinear ODE for $u(t)$,

$$
\frac{d^{3} u}{d t^{3}}=u^{4}
$$

This problem has singular solutions with finite-time blow-up. We use it to introduce the connections between symmetries and stability analysis.

2. Second, a semi-linear heat equation with absorption for $u(x, t)$,

$$
\frac{\partial u}{\partial t}=\frac{\partial^{2} u}{\partial x^{2}}-|u|^{q} u
$$

where $q>0$. The similarity solutions of this problem exhibit a bifurcation from linear to nonlinear spreading behavior depending on the power of the nonlinearity.

3. Finally, blow-up of $u(x, t)$ governed by the Sivashinsky equation,

$$
\frac{\partial u}{\partial t}=-\frac{\partial^{2}}{\partial x^{2}}\left(u^{2}\right)-\sigma \frac{\partial^{2} u}{\partial x^{2}}-\frac{\partial^{4} u}{\partial x^{4}} .
$$

This problem has an infinite number of self-similar solutions as has been observed in singularity formation in other higher-order PDEs. In particular, we show that the stability analysis identifies a unique stable blow-up solution.

These three examples are arranged in order of increasing complexity and are used to represent broader classes of problems.

While similarity solutions for ODEs are often derived via symmetry methods, e.g. [16, $15,59,30]$, the linear stability of these solutions is rarely studied. We describe a method for studying the linear stability of the similarity solutions to (1.1) and use it to argue that generic initial conditions yield solutions that exist only for a finite-time interval, blowing-up both forwards and backwards in time from generic initial data. Such ODEs are also used in the analysis of blow-up in some PDEs [56].

Equation (1.2) is a special case of the general semi-linear heat equation,

$$
u_{t}=\nabla^{2} u+g(u),
$$

which has been studied exhaustively (see [8, 44, 45, 63, 71, 55] and references therein), primarily when $g(u)$ is a positive, nonlinear source term leading to finite-time blow-up. We will concentrate on (1.2) where $g(u)=-|u|^{q} u$ is an absorption term. For this class of equations, global existence is easily established and one can prove the existence of a unique attracting exponentially-localized infinite-time self-similar spreading solution for (1.2) and its generalization to higher dimensions [38, 50, 19]. Different ranges for values of the parameter $q$ yield either diffusion- or absorption-dominated dynamics. This bifurcation was analyzed by Wayne [72] via center-manifold theory, inspired by the renormalization group ideas of Bricmont \& Kupianen [21, 20] and earlier center-manifold ideas of Kohn and collaborators [36, 45] and Bebernes [5]. For $q<0$ equation (1.2) has strong absorption 
that can produce finite-time extinction [39]. More recently the self-similar solutions of (1.2) were studied by Galaktionov \& Williams [41] as part of a hierarchy of absorption-diffusion equations with higher-order diffusion; also see the book by Galaktionov \& Vazquez [40, Chapter 4] for a review of the extensive literature on this problem.

In considering higher-order nonlinear problems, we will apply the stability theory to finite-time blow-up for a PDE in the case of the Sivashinsky equation (1.3) which describes directional solidification [64, 65] or clumping of biological organisms [29]. Blow-up in this model equation was established by Novick-Cohen [57, 58] using an energy method [67]. These results were sharpened and extended by Bernoff \& Bertozzi [10], who studied a related modified Kuramoto-Sivashinsky equation

$$
w_{t}=(1-\alpha) w_{x}^{2} \pm \alpha w_{x x}^{2}-\sigma w_{x x}-w_{x x x x},
$$

for $0<\alpha \leq 1$. For $\alpha=1$ this equation can be transformed to (1.3) by differentiating twice and letting $u=w_{x x}$. Bernoff \& Bertozzi [10] showed that for periodic initial data, depending on the stability of the trivial solution in (1.5), either: (i) if it is unstable, there exists arbitrarily small initial data which lead to finite-time blow-up (ii) if it is stable, there exists a class of finite-amplitude initial data that leads to finite-time blow-up. Numerical results indicated that the solution generically becomes pointwise infinite at a finite time.

Equation (1.3) is also a singular limit of the unstable thin film equation $[13,14,33]$

$$
u_{t}=-\left(u^{m} u_{x}\right)_{x}-\left(u^{n} u_{x x x}\right)_{x}
$$

for the case $m=1, n=0$. This equation generalizes lubrication models of thin films [60]. Note that for $n>0$, there exists solutions of fixed sign, whereas for $n=0$ not only are solutions allowed to change sign, but when a solution blows up, it must change sign (cf. Bertozzi \& Pugh [13] and references therein). Bertozzi \& Pugh conjectured that this equation also manifests finite-time blow-up in the degenerate case when $m+2 \geq n>0$ $[77,48,14,33]$.

Equation (1.3) is also a special case of the Cahn-Hilliard equation, a generalization of the $n=0$ unstable thin film equation (1.6), and is related to other fourth-order nonlinear evolution equations [43]. Blow-up for $m>0$ follows from the earlier work of Levine [53] on a broad set of formally parabolic equations. This connection is described in the review by Evans et al. [35], who also describe extensively the existence of countable sets of similarity solutions for the Cahn-Hilliard equation and its generalizations to higherdimensional axisymmetric problems. Our numerical results on this problem follow the work of Bernoff \& Bertozzi [10] closely, using the ideas described in [73]. Budd and Piggott [23, 24] describe further numerical approaches that are designed to take advantages of symmetries and scale-invariance in computing singular solutions of ODEs and PDEs.

In the next section we analyze similarity solutions and their stability for the ODE problem (1.2). In Section 3, we consider the semi-linear heat equation with absorption (1.3), and use bifurcation theory to examine the transition from diffusively-dominated to absorption dominated spreading solutions at $q=2$. In Section 4, we find a countable infinity of selfsimilar blow-up solutions to the Sivashinsky equation, and use linear stability theory to identify the unique stable profile. A brief discussion and conclusion is given in Section 5 .

bw2008.tex; 23/09/2011; 13:06; p.4 


\section{Self-similar behaviors in an ODE}

Consider the third-order nonlinear ODE for $u(t)$,

$$
\frac{d^{3} u}{d t^{3}}=u^{4}
$$

with initial conditions on $\left(u, u^{\prime}, u^{\prime \prime}\right)$ at some time $t_{0}$. Equations of the form $u^{\prime \prime \prime}=u^{p}$ for $p<0$ have been studied in connection with describing contact lines (where $u \rightarrow 0$ ) of fluid films $[9,17]$; equation (2.1) captures the finite-time blow-up behavior that can occur for $p>1$. The trivial solution $u \equiv 0$ is the only equilibrium, however linearized stability analysis is inconclusive as it is a degenerate fixed point. Note the righthand side of (2.1) is positive definite, and it is easy to show that if all of the initial data are positive then solutions grow without bound and hence $u \equiv 0$ must be an unstable equilibrium. More insight on the behavior expected for general solutions can be obtained by using the symmetries of the equation to find special solutions and to examine their stability.

We look for a scaling symmetry $[4,15,16,59]$, by asking if there exist constants $\mathrm{U}, \mathrm{T}$ such that the ansatz

$$
u(t)=\mathrm{U} \hat{u}(\hat{t}), \quad t=\mathrm{T} \hat{t},
$$

yields the same equation (2.1), applied to $\hat{u}(\hat{t})$, independent of the values of the scaling parameters U, T. This scaling of the dependent and independent variables is often called dimensional analysis when applied to governing equations for physical problems. It is used to recast the problem in terms of dimensionless variables and parameter groups. As described by Barenblatt [4], if dimensional analysis determines relations between all of the scaling constants, then a first-kind similarity solution may exist. Here we find the constraint that $\mathrm{U}=1 / \mathrm{T}$, where $\mathrm{T}$ may be chosen arbitrarily. As the product $t u$ is independent of this parameter, we expect the existence of a solution with $t u=C$ for some constant, $C$. Substituting $u(t)=C / t$ into $(2.1)$ determines $C=-6^{1 / 3}$. We note that $(2.1)$ is an autonomous equation, invariant under time-translations, $t \rightarrow t-t_{c}$ for any critical time $t_{c}$. As such, we can write a one-parameter family of singular solutions,

$$
\bar{u}(t)=-\frac{\sqrt[3]{6}}{t-t_{c}}
$$

This can be written as

$$
\bar{u}(t)=\frac{\sqrt[3]{6}}{t_{c}-t} \rightarrow \infty \quad \text { for }\left(t_{c}-t\right) \searrow 0
$$

namely, the solution is positive for $t<t_{c}$ and blows-up in finite time as $t \rightarrow t_{c}$. In contrast, for $t>t_{c}$ the solution is negative and decays to zero as $t$ increases,

$$
\bar{u}(t)=-\frac{\sqrt[3]{6}}{t-t_{c}} \rightarrow 0 \quad \text { for }\left(t-t_{c}\right) \nearrow \infty
$$


The existence of this one-parameter family of singular solutions of (2.1) is notable, but in order to make stronger statements about whether or not these behaviors are representative of generic nontrivial solutions of (2.1) we must study the stability of solution (2.3).

\subsection{Stability of Self-Similar finite-time BlOW-UP for $t<t_{c}$}

Let us define the time remaining until the blow-up time $\left(t_{c}\right)$ as $\tau \equiv t_{c}-t>0$, and make a change of variables to write general solutions of $(2.1)$ in the form

$$
u(t)=\tau^{-1} U(s), \quad \tau=t_{c}-t, \quad s=-\ln \tau,
$$

where $s$ is a stretched time variable describing evolution of transients for general solutions. Note that $s \rightarrow \infty$ corresponds to $t$ increasing to the critical time $t_{c}$.

Substituting (2.5) into (2.1) yields the autonomous similarity equation for $U(s)$,

$$
\frac{d^{3} U}{d s^{3}}+6 \frac{d^{2} U}{d s^{2}}+11 \frac{d U}{d s}+6 U=U^{4}
$$

The similarity variable ansatz (2.5) has several interpretations. Observing that this choice of $\tau, s$ yields (2.6) as an autonomous equation, ansatz (2.5) can be viewed as a separation of variables for a nonlinear equation. Alternatively, the invariance of (2.6) with respect to the rescaling (2.2) motivates the logarithmic form of $s$, changing the multiplicative scaling to a translational shift, $s=-\ln (T \hat{\tau})=-\ln (\hat{\tau})-\ln T=\hat{s}-\hat{s}_{c}$, where $\hat{s}=-\ln \hat{\tau}$ and $\hat{s}_{c}=-\ln T$.

Self-similar solutions are $s$-independent equilibria of $(2.6): \bar{U}=0$ remains the trivial solution and $\bar{U}=\sqrt[3]{6}$ corresponds to solution (2.4a). Linearizing about the self-similar solution, $U(s) \sim \bar{U}+\epsilon \tilde{U}(s)$ with $\epsilon \ll 1$ yields a linear equation for infinitesimal perturbations to $\bar{U}$,

$$
\frac{d^{3} \tilde{U}}{d s^{3}}+6 \frac{d^{2} \tilde{U}}{d s^{2}}+11 \frac{d \tilde{U}}{d s}+\left(6-4 \bar{U}^{3}\right) \tilde{U}=0 .
$$

Substituting the trial solution $\tilde{U}(s)=e^{\lambda s}$ yields the eigenvalues $\lambda_{1}=1, \lambda_{2,3}=-7 / 2 \pm i \sqrt{23}$ and the general linearized solution

$$
U \sim \bar{U}+\epsilon \tilde{U}(s)=\bar{U}+\underline{c_{1} e^{s}}+c_{2} e^{-7 s / 2} \cos (\sqrt{23} s)+c_{3} e^{-7 s / 2} \sin (\sqrt{23} s) .
$$

The two oscillatory modes decay as $s \rightarrow \infty$. The $\lambda_{1}$ mode has the apparent form of an instability, but as we will demonstrate below it is in fact a symmetry mode, a by-product of the change to similarity variables $(2.5)$ and reflects the exponential divergence in $s$ of the one-parameter family of similarity solutions.

We will demonstrate that the family of solutions with different blow-up times spread exponentially in $s$. The change of variables (2.5) allows us to choose a blow-up time, $t_{c}$. If this value is shifted, $t_{c}=t_{c}^{\prime}+\beta$, then we get

$$
\tau=\tau^{\prime}+\beta=\tau^{\prime}\left(1+\beta / \tau^{\prime}\right)=\tau^{\prime}\left(1+\beta e^{s^{\prime}}\right), \quad s=-\ln \left(\tau^{\prime}+\beta\right)=s^{\prime}-\ln \left(1+\beta e^{s^{\prime}}\right),
$$

and $\beta$ generates a one-parameter family of translated solutions from any solution $U(s)$,

$$
u(t)=\frac{1}{\tau} U(s)=\frac{1}{\tau^{\prime}}\left[\frac{1}{1+\beta e^{s^{\prime}}} U\left(s^{\prime}-\ln \left(1+\beta e^{s^{\prime}}\right)\right)\right]=\frac{1}{\tau^{\prime}} \mathcal{U}\left(s^{\prime}\right)
$$


For the specific case of the blow-up solution, $U(s)=\bar{U}$, we get

$$
\mathcal{U}\left(s^{\prime}\right)=\frac{\bar{U}}{1+\beta e^{s^{\prime}}}=\bar{U}-\underline{\beta \bar{U} e^{s^{\prime}}}+O\left(\beta^{2}\right),
$$

where we have expanded for $\beta \rightarrow 0$, i.e. under the assumption of a small shift in the critical time. Using $s^{\prime} \sim s+O(\beta)$ we can compare the underlined terms in (2.11) and (2.8), showing that the $\lambda_{1}$ mode corresponds to the linearized action of the $t_{c} \rightarrow t_{c}+\beta$ symmetry. Therefore, the presence of the $c_{1}$ term can be re-interpreted as being due to an error in the value of the critical time. The underlined term in (2.8) can always be eliminated by the appropriate shift to $t_{c}$ (here by the estimate $\beta \approx-c_{1} / \bar{U}$ ) to produce the optimal value for $t_{c}$ describing blow-up. The optimal parameter $t_{c}$ selects the solution from the family (2.3) that best approximates the blow-up behavior of a general solution as $u \rightarrow \infty$ [74] for which (2.8) would give the rate of convergence $\left|\left(t_{c}-t\right) u(t)-\bar{U}\right|=O\left(\left[t_{c}-t\right]^{7 / 2}\right)$ as $t \rightarrow t_{c}$. Selecting the wrong blow-up time yields one of the members of the family (2.3) that spreads algebraically in time from the observed blow-up, or in similarity variables a solution that separates exponentially in $s$ from $\bar{U}$. A priori determination of optimal parameters (i.e. prediction of blow-up time) from initial condition is a challenging question for many problems [74].

\subsection{Instability OF SELF-Similar DECAY FOR $t>t_{c}$}

For $t>t_{c}$, it is convenient to describe the decaying solutions in terms of the variables

$$
u(t)=\check{\tau}^{-1} \check{U}(\check{s}), \quad \check{\tau}=t-t_{c}, \quad \check{s}=\ln \check{\tau},
$$

where $\check{s}, \check{\tau} \rightarrow \infty$ as $t \rightarrow \infty$. This yields an equation comparable to (2.6),

$$
\frac{d^{3} \check{U}}{d \check{s}^{3}}-6 \frac{d^{2} \check{U}}{d \check{s}^{2}}+11 \frac{d \check{U}}{d \check{s}}-6 \check{U}=\check{U}^{4}
$$

The nontrivial fixed point of this equation is $\bar{U}=-\sqrt[3]{6}$ corresponding to the self-similar solution $(2.4 \mathrm{~b})$. Proceeding with the linear stability analysis of this fixed point yields $\check{\lambda}_{1}=$ $-1, \check{\lambda}_{2,3}=7 / 2 \pm i \sqrt{23}$

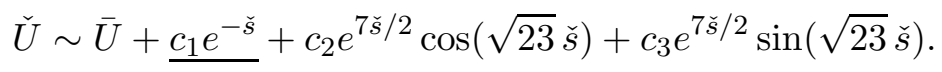

We can again identify the first eigenmode as a symmetry mode, associated with the action of the shift of the parameter $t_{c}=t_{c}^{\prime}+\check{\beta}$. Analogous to (2.10), this now yields a one parameter family of solutions

$$
u(t)=\frac{1}{\check{\tau}^{\prime}}\left[\frac{1}{1-\check{\beta} e^{-\check{s}^{\prime}}} \check{U}\left(\check{s}^{\prime}+\ln \left(1-\check{\beta} e^{-\check{s}^{\prime}}\right)\right)\right]=\frac{1}{\check{\tau}^{\prime}} \check{\mathcal{U}}\left(\check{s}^{\prime}\right) .
$$

When this time-shift is applied to the self-similar solution, we obtain

$$
\check{\mathcal{U}}\left(\check{s}^{\prime}\right)=\frac{\bar{U}}{1-\check{\beta} e^{-\check{s}^{\prime}}}=\bar{U}+\underline{\check{\beta}} \bar{U} e^{-\check{s}^{\prime}}+O\left(\check{\beta}^{2}\right) .
$$




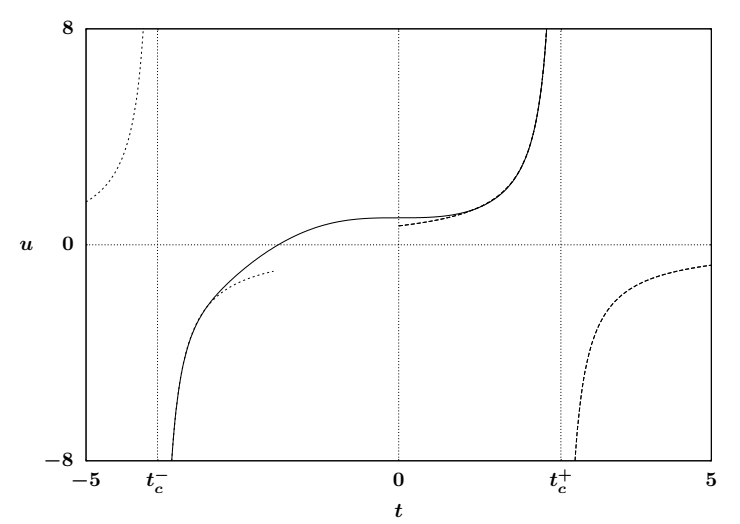

Figure 1. Computed solution (solid curve) of (2.1) starting from initial data at $t_{0}=0$ compared with the optimal self-similar solutions at $t_{c}^{-}, t_{c}^{+}$(dashed curves).

Here the consequence of an arbitrary choice of $t_{c}$ is a decaying perturbation corresponding to $\lambda_{1}=-1$. This can be expected since the error becomes small as $t \rightarrow \infty$ and the influence of $t_{c}$ fades into the past. However, the other modes have $\operatorname{Re}\left(\check{\lambda}_{2,3}\right)>0$ and represent genuine instabilities so the algebraically decaying behavior described by $(2.4 \mathrm{~b})$ is unstable to perturbations for $t \rightarrow \infty$.

\subsection{Global Behavior}

We are now in position to make statements about the behavior of solutions of (2.1) starting from generic initial data at some time $t_{0}$. The trivial and infinite-time decay solutions are unstable, so those behaviors will not commonly occur. In fact, the self-similar decaying solution traces out a one-dimensional manifold of solutions attracted to the origin. Solutions from all other initial conditions will be attracted to the stable self-similar finite-time blow-up given by $(2.4 \mathrm{a})$ for some $t_{c}^{+}>t_{0}$.

We may also inquire about the behavior backward in time, for $t<t_{0}$. Noting that (2.1) is invariant under the reflection symmetry, $u \rightarrow-u$ and $t \rightarrow-t$, our stability results also apply backward in time. Namely, as $t$ decreases from $t_{0}$, generic solutions will blow-up with $u \rightarrow-\infty$ as $t$ decreases towards some $t_{c}^{-}$; only solutions given exactly by $(2.4 \mathrm{~b})$ will exist for all earlier times, $-\infty<t<t_{0}$.

Fig. 1 shows a computed solution on $t_{c}^{-}<t<t_{c}^{+}$, starting from initial data $\left(u, u^{\prime}, u^{\prime \prime}\right)=$ $(1,0,0)$ at $t_{0}=0$ with the optimal similarity solutions that give the asymptotics of its blow-up behaviors at $t_{c}^{-} \approx-3.856$ and $t_{c}^{+} \approx 2.592$. These values for $t_{c}$ can be estimated from (2.3) in terms of a general solution of (2.1) if $|u(t)|$ is large, $u\left(t_{*}\right)=u_{*}$ yielding $t_{c} \approx t_{*}+\sqrt[3]{6} / u_{*}$. For solutions with initial conditions lying in a neighborhood close to $(2.3)$, we can observe the spiral transient structure predicted by (2.8). Fig. 2(left) shows solutions in a scaled phase-space $\left(u^{3}, u^{\prime} u, u^{\prime \prime}\right)$ motivated by the scaling $(2.2)$ in that all three axes scale like $O\left(\mathrm{U}^{3}\right)$. In this representation, all of the singular solutions (2.3) lie on a single diagonal line,

$$
\left(\bar{u}^{3}, \bar{u}^{\prime} \bar{u}, \bar{u}^{\prime \prime}\right)=\left(6^{2 / 3} \theta, 6^{1 / 3} \theta, 2 \theta\right) \quad-\infty<\theta<\infty .
$$



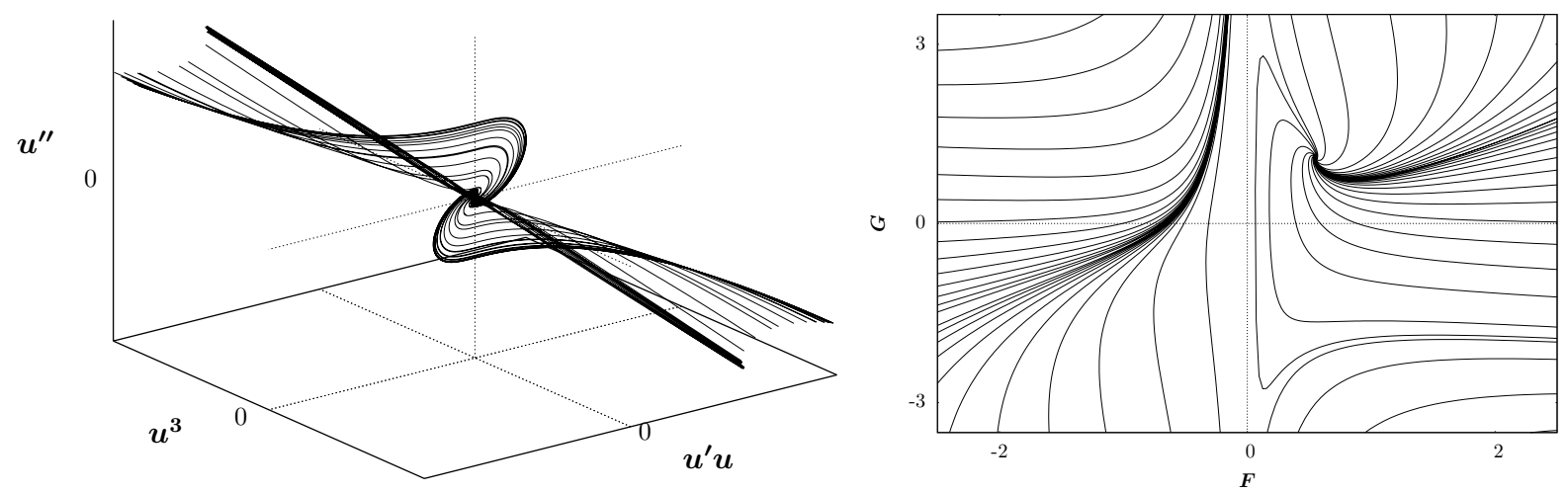

Figure 2. (left) A 3-D phase-space representation of solutions starting from perturbations of (2.3) at $t=0$. Solutions eventually converge back to the manifold of singular solutions (2.17) (the diagonal line) for $|u| \rightarrow \infty$. (right) Phase plane representation (2.18) of solutions of (2.1).

Generic trajectories will approach the diagonal as $t \rightarrow \pm \infty$ for which $u \rightarrow \pm \infty$.

Finally, recall that an autonomous ODE of degree $n$ with a scale invariance can be reduced by a change of both independent and dependent variables to a system of degree $n-1$ $[15,16,59]$. Equation (2.17) suggests two scale-invariant variables, $F=u^{\prime} / u^{2}, G=u^{\prime \prime} /\left(u^{\prime} u\right)$, and the rescaling symmetry motivates the independent variable $\zeta=\ln |u|$ which transforms the rescaling symmetry in $u$ into a translational symmetry in $\zeta$. This reduces $(2.3)$ to an autonomous system [17],

$$
\frac{d F}{d \zeta}=G-2 F, \quad \frac{d G}{d \zeta}=2(G-2 F)+\frac{1-F(G-2 F)^{2}-7 F^{2}(G-2 F)-6 F^{3}}{F^{2}}
$$

whose phase plane is graphed in Fig. 2(right). One can analyze this system with standard phase plane methods, however caution is needed for solutions passing through $u=0$ as $F, G$ and $\zeta$ all become singular. Here solution (2.3) is represented by the fixed point $(F, G)=$ $\left(6^{-1 / 3}, 2 \cdot 6^{-1 / 3}\right)$, see Fig. 2(right). Linear stability analysis shows this fixed point to be a stable spiral. Note that when $|u|$ increases, so does $\zeta$ and the linear stability of the fixed point in effect reproduces the stability analysis of (2.7); the reduction to the second-order system has eliminated the $c_{1}$ symmetry mode in (2.8). Note that $|u| \rightarrow 0$ corresponds to $\zeta \rightarrow-\infty$, hence decaying behavior of $u(t)$ maps onto time-reversed trajectories in the phase plane, with $\zeta \rightarrow-\zeta$. Consequently the spiral fixed point also describes the instability analyzed in $(2.14)$.

\section{Self-similar spreading in a semi-linear heat equation with absorption}

We consider positive solutions of the semi-linear heat equation with absorption for a temperature field $u(x, t)$,

$$
u_{t}=u_{x x}-|u|^{q} u
$$


for $q>0$ with finite-mass non-negative initial data on the real line, $u(x, 0)=I(x)$, that decays exponentially in the far-field. These initial conditions become uniformly positive for $t>0$ due to the infinite speed of propagation of diffusion. As such, we can replace $|u|^{q} u$ with $u^{q+1}$ in (3.1), yielding

$$
u_{t}=u_{x x}-u^{q+1}
$$

which will be used for the remainder of this section.

Physically, $u(x, t)$ can be interpreted as a dimensionless temperature in a system subject to linear diffusion with a non-linear absorption term modeling the loss of heat; typically $u(x, t)$ is measured relative to the external ambient temperature and the heat loss is a powerlaw determined by the geometry or the physics (for example Stefan's law of cooling which would yield $q=3$ ). We will show that the competition between diffusion and absorption determines the asymptotic behavior of the solution. We give a heuristic argument for the asymptotic behavior of the solution and then show how to quantify our predictions using similarity variables.

This equation has a maximum principle; at the maximum of $u(x, t)$ diffusion can only act to reduce the amplitude of the solution. As such, the absorption term can be used to prove a decay bound [41]. If we define $u_{\max }(t)=\max _{x} u(x, t)$ then $u_{\max }(t)$ is bounded by the solution of $d u / d t=-u^{q+1}$, namely

$$
u_{\max }(t) \leq \frac{u_{\max }(0)}{\left(1+q\left[u_{\max }(0)\right]^{q} t\right)^{1 / q}} .
$$

Hence solutions decay like $O\left(t^{-1 / q}\right)$ as $t \rightarrow \infty$ for $q>0$.

In the absence of the absorption term, equation (3.2) is just the heat equation $u_{t}=u_{x x}$; exponentially-localized, finite-mass initial conditions will approach a Gaussian solution at large times,

$$
u \sim \bar{u}(x, t) \equiv \frac{m_{c}}{\sqrt{4 \pi\left(t-t_{c}\right)}} \exp \left(-\frac{\left(x-x_{c}\right)^{2}}{4\left(t-t_{c}\right)}\right) \quad t \rightarrow \infty,
$$

where $m_{c}$ is the mass of the solution, $x_{c}$ is the center of mass, and $t_{c}$ can be determined from the variance of the initial condition. The key feature to note in this solution is that the amplitude decays like $O\left(t^{-1 / 2}\right)$.

Both absorption and diffusion decrease the solution's amplitude. As seen in (3.3), absorption scales the amplitude of the solution proportional to $t^{-1 / q}$ while diffusion does so proportional to $t^{-1 / 2}$. The mechanism that reduces the temperature most rapidly will dominate (see the discussions in $[38,50,19,20,72,41])$. Specifically:

- For $q>2$ diffusion dominates and the solution approaches a Gaussian (3.4) whose amplitude decays like $t^{-1 / 2}$ as $t \rightarrow \infty$.

- For $0<q<2$ absorption dominates and the solution approaches a non-Gaussian selfsimilar decaying profile, whose amplitude scales like the absorption bound (3.3), $t^{-1 / q}$ as $t \rightarrow \infty$. 
- For the critical case of $q=2$ the rates of absorption and diffusion coincide. The solution approaches a Gaussian profile but the amplitude's decay rate has acquired a logarithmic correction, $(t \ln t)^{-1 / 2}$ as $t \rightarrow \infty$.

Following the approach of Wayne [72], we will show how to associate this change of behavior with a bifurcation in $q$ of the trivial solution in similarity variables.

\subsection{Similarity VARiables}

We apply the dimensional scaling,

$$
u(x, t)=\mathrm{U} \hat{u}(\hat{x}, \hat{t}), \quad t=\mathrm{T} \hat{t}, \quad x=\mathrm{L} \hat{x},
$$

transforming (3.2) to

$$
\left[\frac{1}{\mathrm{~T}}\right] \hat{u}_{\hat{t}}=\left[\frac{1}{\mathrm{~L}^{2}}\right] \hat{u}_{\hat{x} \hat{x}}-\left[\mathrm{U}^{q}\right] \hat{u}^{q+1} .
$$

Scale-invariance determines $\mathrm{U}=\mathrm{T}^{-1 / q}$ and $\mathrm{L}=\mathrm{T}^{1 / 2}$, namely the amplitude decays at a rate dependent on $q$ while the solution spreads diffusively for $\mathrm{T} \rightarrow \infty$. Consequently, we write solutions of (3.2) in terms of similarity variables as

$$
u(x, t)=\tau^{-1 / q} U(\eta, s), \quad \tau=t-t_{c}, \quad \eta=\frac{x-x_{c}}{\sqrt{\tau}}, \quad s=\ln \tau,
$$

where $x_{c}, t_{c}$ are constants. This yields the similarity PDE for $U(\eta, s)$,

$$
U_{s}=\frac{1}{q} U+\frac{1}{2} \eta U_{\eta}+U_{\eta \eta}-U^{q+1},
$$

with boundary conditions that $U$ vanish exponentially in the far-field, $U(|\eta| \rightarrow \infty) \rightarrow 0$. These boundary conditions are motivated by the exponentially localized initial conditions considered earlier. If one relaxes these conditions, there exist a one-parameter family of selfsimilar solutions with algebraic decay in the far-field, some with finite mass and some with infinite mass [19, 20, 38]; these solutions will not be discussed here. Self-similar solutions are $s$-independent steady states of this problem, $U=\bar{U}(\eta)$,

$$
0=\frac{1}{q} \bar{U}+\frac{1}{2} \eta \bar{U}_{\eta}+\bar{U}_{\eta \eta}-\bar{U}^{q+1} .
$$

Linearizing (3.8) about the self-similar solutions, $U(\eta, s) \sim \bar{U}(\eta)+\epsilon \tilde{U}(\eta, s)$, yields the equation

$$
\tilde{U}_{s}=\mathcal{L} \tilde{U}, \quad \mathcal{L} \tilde{U} \equiv \frac{1}{q} \tilde{U}+\frac{1}{2} \eta \tilde{U}_{\eta}+\tilde{U}_{\eta \eta}-(q+1) \bar{U}^{q} \tilde{U}
$$

which can be solved via separation of variables, $\tilde{U}(\eta, s)=\phi(\eta) e^{\lambda s}$, to yield the eigenvalue problem for the linear stability of $\bar{U}(\eta)$,

$$
\lambda \phi=\mathcal{L} \phi,
$$

where $\phi$ decays exponentially as $\eta \rightarrow \pm \infty$. 


\subsection{Diffusion-Dominated SPREAding Via Stability of the trivial SOlution}

For the stability of the trivial solution, $\bar{U}(\eta) \equiv 0,(3.11)$ reduces to

$$
\lambda \phi=\frac{1}{q} \phi+\frac{1}{2} \eta \phi_{\eta}+\phi_{\eta \eta}
$$

This is a shifted Hermite operator yielding the discrete set of eigensolutions,

$$
\phi_{k}(\eta)=H_{k}(\eta / 2) e^{-\eta^{2} / 4}, \quad \lambda_{k}=\frac{1}{q}-\frac{k+1}{2}, \quad k=0,1,2, \cdots,
$$

where $H_{k}(y)$ are the Hermite polynomials, $H_{0}(y)=1, H_{1}(y)=2 y, H_{2}(y)=4 y^{2}-2, \cdots$. The largest eigenvalue is $\lambda_{0}=\frac{1}{q}-\frac{1}{2}$, from which we see that the trivial solution changes stability at $q=2$. All of the eigenvalues are negative for $q>2$ hence we expect the trivial solution to be stable, and the long-time behavior to be given by the slowest decaying eigenmode,

$$
U \approx \tilde{U}(\eta, s)=\sum_{k=0}^{\infty} a_{k} \phi_{k}(\eta) e^{\lambda_{k} s} \sim a_{0} \phi_{0}(\eta) e^{\lambda_{0} s} .
$$

Noting that $\tau^{-1 / q} e^{\lambda_{0} s}=\left(t-t_{c}\right)^{-1 / 2}$, written in original variables, the first term in the series yields the Gaussian solution (3.4) with $m_{c}=a_{0} \sqrt{4 \pi}$ being the "effective mass" of this solution at large times. ${ }^{1}$ Solution (3.4) was shown to be a global attractor for (3.2) for $q>2$ with finite-mass exponentially localized initial conditions [38]. Note that the second term in the expansion, $\phi_{1}(\eta) e^{\lambda_{1} s}$, yields a correction of $O\left(\left(t-t_{c}\right)^{-1}\right)$ to (3.4), but there may also be larger nonlinear contributions to the error as we show below.

This solution may seem surprising in that it has fixed mass even in the presence of the absorption term; for $q>2$ only a finite proportion of the initial mass is lost as $t \rightarrow \infty$. Consider the integral of the solution in similarity variables,

$$
M(s)=\int_{-\infty}^{\infty} U(\eta, s) d \eta>0 .
$$

In terms of $M,(3.8)$ yields

$$
\frac{d M}{d s}=\lambda_{0} M-\int_{-\infty}^{\infty} U^{q+1} d \eta
$$

For $q>2, \lambda_{0}<0$ and $M(s)$ decays to zero, with the linear term controlling the large-time behavior of the solution. Assuming the behavior $U(\eta, s) \sim a_{0} \phi_{0}(\eta) e^{\lambda_{0} s}$ in (3.16) yields the asymptotic expansion

$$
M(s) \sim m_{c} e^{\lambda_{0} s}+O\left(e^{(q+1) \lambda_{0} s}\right) \quad \text { as } \quad s \rightarrow \infty,
$$

where the error term comes from the nonlinear absorption. We see that the first term from (3.17) corresponds to (3.4) and we can now combine the error terms from the linear and nonlinear estimates to yield $O\left(\left[t-t_{c}\right]^{-\min (1,(q-1) / 2)}\right)$ as $t \rightarrow \infty$.

\footnotetext{
${ }^{1}$ In fact, since $\lambda_{k}-1 / q=-(k+1) / 2$, series (3.14) yields $\tilde{u}=\sum_{k} a_{k} \phi_{k}(\eta)\left(t-t_{c}\right)^{-(k+1) / 2}$.
} 
In fact, solution (3.4) for $q>2$ could also be predicted from the dimensional analysis in (3.6), i.e. another choice for the scalings exists. As before, $\mathrm{L}^{2}=\mathrm{T}$, but if $\mathrm{U}^{q}<1 / \mathrm{T}$ for $\mathrm{T} \rightarrow$ $\infty$ then the absorption term is sub-dominant and would not play a role in determining the similarity solution, reducing (3.2) to the heat equation, $u_{t}=u_{x x}$. The heat equation's large time asymptotics are well studied; see for example Wayne [72] or the discussion in Witelski $\&$ Bernoff [74] based on earlier works [78, 51, 52]. Solutions of the heat equation on the real line conserve the integral $\int u d x=\mathrm{UL} \int \hat{u} d \hat{x}$, so $\mathrm{U}$ is determined by $\mathrm{U}=1 / \mathrm{L}=\mathrm{T}^{-1 / 2}$. This gives $\mathrm{U}^{q}=\mathrm{T}^{-q / 2}$ so if $q>2$ then $\mathrm{U}^{q} \ll 1 / \mathrm{T}$ for $\mathrm{T} \rightarrow \infty$, consistent with the conclusion based on the stability analysis.

Solutions like (3.4) determined by a dominant balance in which not all of the terms from the governing equation are present are sometimes called asymptotically self-similar solutions. This is in-line with Barenblatt's description of self-similar solutions serving as intermediate asymptotic states for $t \rightarrow \infty$ [3, p. 171].

For $q>2$, we have established that at large times the solution to the semi-linear heat equation with absorption is diffusion-dominated and approaches the Gaussian solution (3.4), familiar to us as the Green's function for the heat equation.

Finally, we note that there are three free parameters in the Gaussian (3.4): the mass $\left(m_{c}\right)$, the center of mass $\left(x_{c}\right)$, and the critical time associated with the variance of the solution $\left(t_{c}\right)$. A solution of (3.2) for $q>2$ starting from any appropriate initial data will be asymptotic to the Gaussian for some choice of these parameters. Since mass is not conserved for all times, it is nontrivial to select $m_{c}$ to ensure that $|u(x, t)-\bar{u}(x, t)| \ll O\left(t^{-1 / 2}\right)$ as $t \rightarrow \infty[3$, p. 152]. Using the ideas of optimal asymptotics [51, 74] one can in principle choose parameters $x_{c}, t_{c}$ to eliminate the contributions to the error term from the $\lambda_{1}, \lambda_{2}$ eigenmodes, to reduce the linear error term in (3.14) from $O\left(\left[t-t_{c}\right]^{-1}\right)$ to $O\left(\left[t-t_{c}\right]^{-2}\right)$. The nonlinear error from (3.17) will persist independent of these choices.

\subsection{BifURCATION TO NONLINEAR SPREADING}

As $q$ decreases through 2, the zero state loses stability to a branch of nonlinear solutions. To describe this bifurcating branch of solutions, we perform a center manifold expansion $[72,27,46]$.

It is convenient to use the $L^{2}$ inner-product, $\langle v, w\rangle=\int_{-\infty}^{\infty} v w d \eta$, to project perturbations onto the space of eigenfunctions. Then the adjoint problem to (3.12) is

$$
\lambda \psi=\frac{1}{q} \psi-\frac{1}{2} \psi-\frac{1}{2} \eta \psi_{\eta}+\psi_{\eta \eta}, \quad \psi(|\eta| \rightarrow \infty) \rightarrow 0,
$$

yielding adjoint eigenfunctions $\psi_{k}(\eta)=H_{k}(\eta / 2)$. If $q=2+\epsilon$, then $\lambda_{0}=-\epsilon / 4+O\left(\epsilon^{2}\right)$ so solutions evolve slowly. Consequently, we can do a weakly-nonlinear analysis for $\epsilon \rightarrow$ 0 . Taking $U(\eta, s) \sim A_{0}(s) \phi_{0}(\eta)$ and projecting (3.8) onto $\psi_{0}$ at leading order yields the amplitude equation

$$
\frac{d A_{0}}{d s}=\lambda_{0} A_{0}-\frac{A_{0}^{3}}{\sqrt{3}}
$$


This equation has two branches of steady solutions,

$$
\bar{A}_{0}=0, \quad \text { and } \quad \bar{A}_{0}=\left(\frac{(2-q) \sqrt{3}}{2 q}\right)^{1 / 2} \quad \text { for } q<2 .
$$

This is a supercritical pitchfork bifurcation; for $q>2$, the zero state is stable, as shown in the previous section. For $q<2$ there is a change of stability to the nontrivial branch. In terms of original variables, this yields the similarity solutions for $q \nearrow 2$,

$$
u(x, t) \approx\left(\frac{(2-q) \sqrt{3}}{2 q}\right)^{1 / 2} \tau^{-1 / q} e^{-\eta^{2} / 4} .
$$

For the critical case $q=2, \lambda_{0}=0$ yielding a degenerate case for (3.19). The solution $A_{0}=0$ is now nonlinearly stable; solving for $A_{0}(s)$ yields $A_{0}(s)=(\sqrt{3} /(2 s+C))^{1 / 2}$, corresponding to asymptotic behavior for $t \rightarrow \infty$

$$
u(x, t) \approx(3 / 4)^{1 / 4}[\tau \ln \tau]^{-1 / 2} e^{-\eta^{2} / 4} .
$$

The logarithmic dependence on $\tau$ here reflects the fact that in the critical case $A_{0}$ is decaying only algebraically, as opposed to exponentially, with respect to $s$.

\subsection{The BRANCH OF POSITIVE SELF-SIMILAR SOlUtions FOR $0<q<2$}

Self-similar solutions of (3.2) for $0<q<2$ are nontrivial solutions of equation (3.9),

$$
\frac{1}{q} \bar{U}+\frac{1}{2} \eta \bar{U}_{\eta}+\bar{U}_{\eta \eta}-\bar{U}^{q+1}=0,
$$

with $\bar{U} \rightarrow 0$ exponentially as $|\eta| \rightarrow \infty$. This problem has a unique branch of positive finite-mass solutions on $0<q<2$. We note that additional branches of unstable solutions bifurcate from the zero-state at $q=2 /(k+1)$ for $k=1,2, \cdots$ (that is when $\lambda_{k}=0$ ); these solutions have sign-changes and are not considered here [41].

There are no closed-form analytic $\bar{U}(\eta)$, so the solutions for this problem must be computed numerically. Seeking symmetric solutions, we impose $\bar{U}_{\eta}(0)=0$ and compute for positive $\eta$. One possible approach is a shooting method starting from $\eta=0$ with the value for $\bar{U}(0)$ as a shooting parameter. Alternatively, a global relaxation method based on a Newton-Raphson fixed-point iteration for a finite-difference discretization of the boundary value problem for (3.9) can be implemented [73]. To carry out the computation on a large but finite domain, we need to determine an appropriate far-field boundary condition; this can be done by applying WKB analysis to (3.9) linearized about $\bar{U}=0$ yielding $[20,50]$

$$
\bar{U}(\eta) \sim C|\eta|^{-1+2 / q} e^{-\eta^{2} / 4} \quad|\eta| \rightarrow \infty .
$$

This estimate is a non-monotone function, but the condition $|\eta| \gg \sqrt{[4-2 q] / q}$ ensures a sufficiently large computational domain so that it captures the form of the solution and leads to a reliable homogeneous Robin boundary condition,

$$
\frac{d \bar{U}}{d \eta}+\left(\frac{1}{2} \eta+\frac{q-2}{q \eta}\right) \bar{U}=0 \quad \text { as } \quad|\eta| \rightarrow \infty .
$$

bw2008.tex; 23/09/2011; 13:06; p.14 

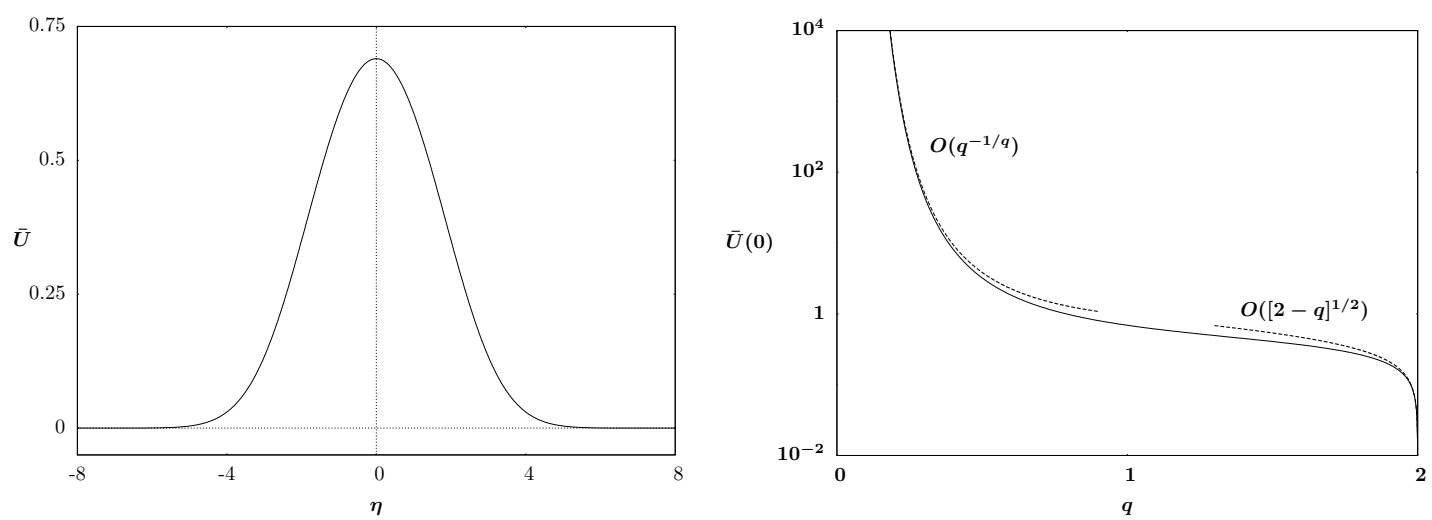

Figure 3. (left) The numerical computed self-similar solution $\bar{U}(\eta)$ for $q=1$ and (right) the branch of positive self-similar solutions on $0<q<2$ with the asymptotes for $q \rightarrow 0$ and $q \rightarrow 2$, (3.20).

See Fig. 3 (left) for the solution for $q=1$, with solutions for other values of $q$ having the same qualitative form.

As $q$ decreases to zero, the amplitude of the solutions increases monotonically. For the limit $q \rightarrow 0$ we follow the analysis in [41] in rescaling the solution by $\bar{U}(\eta)=q^{-1 / q} \bar{V}(\eta)$ and expanding $|\bar{V}|^{q} \sim 1+q \ln |\bar{V}|$ to obtain the leading order equation for the solution profile,

$$
\frac{1}{2} \eta \bar{V}_{\eta}+\bar{V}_{\eta \eta}-\bar{V} \ln |\bar{V}|=0
$$

Solution of this problem via shooting suggests $\bar{V}(0) \approx 0.963$ and gives a good estimate of the amplitude of the positive self-similar solutions for $q \rightarrow 0$, see Fig. 3 (right).

\subsection{LineAR StABILITY OF THE POSITIVE SELF-SIMILAR SOLUTIONS FOR $0<q<2$}

Having obtained the solutions $\bar{U}(\eta)$ in numerical form, we can similarly numerically solve the stability problem (3.11) to obtain some of the largest eigenvalues in the spectrum [73]. Analogous to (3.24), applying WKB to $\mathcal{L} \phi=\lambda \phi$ gives us convenient far-field boundary conditions for the eigenfunctions,

$$
\frac{d \phi}{d \eta}+\left(\frac{1}{2} \eta+\frac{q-2}{q \eta}\right) \phi=-\frac{2 \lambda}{\eta} \phi \quad|\eta| \rightarrow \infty .
$$

The discretized problem is now a matrix eigenvalue/eigenvector problem and can be solved using inverse iteration or other methods from numerical linear algebra. Important insight is gained by comparing these computed results against the analytical predictions, see Fig. 4.

For $q \rightarrow 2$, we can use the center-manifold analysis from $\S 3.3$ to deduce the spectrum. The leading order behavior of the largest eigenvalue is governed by the linearization of (3.19) around the steady state on the center manifold,

$$
\lambda_{0}(q) \sim \frac{2-q}{2 q}-\frac{3}{\sqrt{3}} \bar{A}_{0}^{2}=-\frac{2-q}{2},
$$




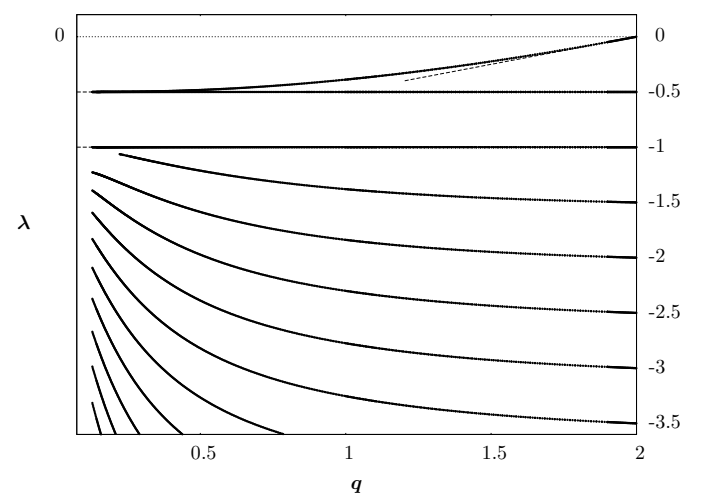

Figure 4. Numerically computed eigenvalues (solid curves) for the stability of the positive self-similar solution $\bar{U}(\eta)$ on $0<q \leq 2$. The two $q$-independent symmetry modes $\lambda=-\frac{1}{2},-1$ are confirmed along with analytical predictions for the other modes for $q \rightarrow 2$ (dashed curves) $(3.27,3.28$ ).

while the eigenvalues associated with stable manifold remain constant to leading order for $q \rightarrow 2$,

$$
\lambda_{k}(q) \sim-k / 2 \quad k=1,2, \cdots .
$$

Two of the eigenmodes are consequences of the invariance of the original PDE with respect to shifts in $x$ or $t$ and the form of the similarity variables (3.7). As in $\S 2.1$, if the constant $t_{c}$ is subject to a shift, $t_{c}=t_{c}^{\prime}+\beta$, one solution of (3.2) is transformed to another, and in terms of similarity variables, when applied to a self-similar solution, takes the form

$$
u(x, t)=\left(\tau^{\prime}\right)^{-1 / q}\left[\left(1-\beta e^{-s^{\prime}}\right)^{-1 / q} \bar{U}\left(\eta^{\prime}\left(1-\beta e^{-s^{\prime}}\right)^{-1 / 2}\right)\right]=\left(\tau^{\prime}\right)^{-1 / q} \mathcal{U}\left(\eta^{\prime}, s^{\prime}\right) .
$$

Expanding $\mathcal{U}$ for $\beta \rightarrow 0$ yields

$$
\mathcal{U}\left(\eta^{\prime}, s^{\prime}\right) \sim \bar{U}\left(\eta^{\prime}\right)+\beta\left(\frac{1}{q} \bar{U}+\frac{1}{2} \eta^{\prime} \frac{d \bar{U}}{d \eta}\right) e^{-s^{\prime}}+O\left(\beta^{2}\right),
$$

yielding the eigenmode connected with the $t_{c} \rightarrow t_{c}+\beta$ symmetry for all $q$,

$$
\phi(\eta)=\frac{1}{q} \bar{U}+\frac{1}{2} \eta \frac{d \bar{U}}{d \eta}, \quad \lambda=-1 .
$$

Similarly, for shifts of $x_{c}=x_{c}^{\prime}+\gamma$ we obtain

$$
u(x, t)=\tau^{-1 / q} \bar{U}\left(\eta^{\prime}-\gamma e^{-s / 2}\right)=\tau^{-1 / q} \mathcal{U}\left(\eta^{\prime}, s\right) .
$$

Expanding $\mathcal{U}$ for $\gamma \rightarrow 0$ yields

$$
\mathcal{U}\left(\eta^{\prime}, s\right) \sim \bar{U}-\gamma \frac{d \bar{U}}{d \eta} e^{-s / 2}+O\left(\gamma^{2}\right)
$$

hence the eigenmode corresponding to spatial translation of $x_{c}$ for all $q$ is

$$
\phi(\eta)=\frac{d \bar{U}}{d \eta}, \quad \lambda=-\frac{1}{2} .
$$




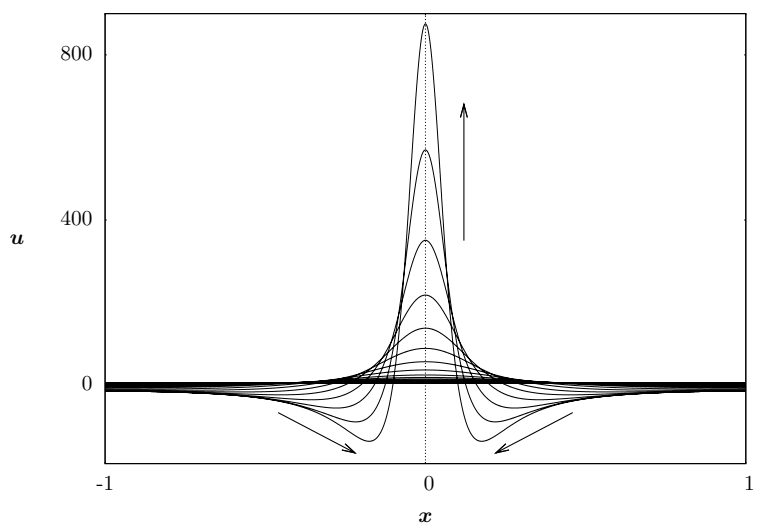

Figure 5. Numerical simulation of an initial value problem for (4.3) with Neumann boundary conditions on $-1 \leq x \leq 1$.

We have now identified the two eigenvalues $\lambda=-\frac{1}{2}$ and $\lambda=-1$ with translations in space and time. One could again remove the projections onto these modes at large time by optimal-asymptotic choices of $x_{c}$ and $t_{c}$. However in this case there is little to be gained because as seen in Fig. 4 the largest eigenvalue, $\lambda_{0}(q)$, is greater that $-\frac{1}{2}$ and not associated with the symmetry modes. Unlike the $q>2$ case, here there is no continuous family of solutions with respect to $m_{c}$ for arbitrary mass. The integral $\bar{M}(q)=\int \bar{U} d \eta$ is uniquely defined for each $q$ and scales like $\bar{U}(0 ; q)$, see Fig. 3 (right). As such, we expect that for $q<2$ as $t \rightarrow \infty$

$$
u(x, t) \sim \tau^{-1 / q} \bar{U}(\eta)+O\left(\tau^{-1 / q+\lambda_{0}(q)}\right),
$$

where $\tau=t-t_{c}$ and $\eta=\left(x-x_{c}\right) / \sqrt{\tau}$ for any choice of $x_{c}$ and $t_{c}$. In original variables, the mass, $m=\int u d x$, of these solutions decays like $O\left(\tau^{1 / 2-1 / q}\right)$.

\section{Self-similar finite-time blow-up in a higher-order PDE}

We now consider the dynamics of the Sivashinsky equation,

$$
u_{t}=-\left(u^{2}\right)_{x x}-\sigma u_{x x}-u_{x x x x} .
$$

It is well known that this equation manifests self-similar blow-up [57, 58, 10, 13, 35] in which $|u(x, t)|$ becomes infinite at a point in finite time; here we show that it has multiple self-similar blow-up solutions and analyze their stability. Analysis of finite-time blow-up and rupture in many higher order PDEs starts along similar lines [35, 11, 76, 77, 79, 34, 22, 25].

Using the non-dimensionalization (3.5) in (4.1) yields

$$
\left[\frac{1}{\mathrm{~T}}\right] \hat{u}_{\hat{t}}=-\left[\frac{\mathrm{U}}{\mathrm{L}^{2}}\right]\left(\hat{u}^{2}\right)_{\hat{x} \hat{x}}-\sigma\left[\frac{1}{\mathrm{~L}^{2}}\right] \hat{u}_{\hat{x} \hat{x}}-\left[\frac{1}{\mathrm{~L}^{4}}\right] \hat{u}_{\hat{x} \hat{x} \hat{x} \hat{x}} .
$$


Finite-time blow-up behavior implies that $\mathrm{U} \rightarrow \infty$ at a finite critical time $t_{c}$; as such, $\mathrm{T}$ scales as the remaining time till blow-up and we will study the scaling behavior near blow-up, namely as $\mathrm{T} \rightarrow 0$. Examining the two second-order terms suggests that the linear term, $\sigma u_{x x}$, will be negligible compared to the nonlinear term, $\left(u^{2}\right)_{x x}$, in the limit of blowup. Hence, while the linear diffusive term plays a role in determining the existence and stability of steady states, it is sub-dominant in the asymptotic limit of self-similar blowup. Consequently the reduced form of (4.1) that yields the invariant scalings $U=T^{-1 / 2}$, $\mathrm{L}=\mathrm{T}^{1 / 4}$ is

$$
u_{t}=-\left(u^{2}\right)_{x x}-u_{x x x x} .
$$

The similarity solutions of (4.3) are the asymptotically self-similar solutions of (4.1). This finite-time behavior corresponding to $\mathrm{T} \rightarrow 0$ with the solution blowing up $(\mathrm{U} \rightarrow \infty)$, and the lengthscale vanishing $(\mathrm{L} \rightarrow 0)$, describes so-called focusing singularities.

Fig. 5 shows a numerical simulation of (4.3) on $-1 \leq x \leq 1$ with Neumann boundary conditions and initial data $u(x, 0)=5+\cos (\pi x) / 10$. The results are suggestive of finitetime blow-up at $x_{c}=0$. Not all initial conditions for the Neumann problem for (4.3) lead to blow-up; Bernoff \& Bertozzi [10] classified which initial data lead to constant steady solutions vs. finite-time blow-up using a combination of bifurcation theory and some ideas due to Palais [61]. We will demonstrate below that this simulation converges to a self-similar blow-up solution.

\subsection{Similarity VARIABles}

The above scalings motivate the choice of similarity variables [10, 35],

$$
u(x, t)=\tau^{-1 / 2} U(\eta, s), \quad \tau=t_{c}-t, \quad \eta=\left(x-x_{c}\right) / \tau^{1 / 4}, \quad s=-\ln \tau,
$$

where $x_{c}, t_{c}$ are constants giving the blow-up position and time. Self-similar solutions are $s$-independent solutions, $U(\eta, s)=\bar{U}(\eta)$.

Returning to the simulation shown in Fig. 5, one can ask how to empirically show that self-similar dynamics are being approached without knowing $x_{c}, t_{c}$ a priori. A simple estimate of the blow-up position $x_{c}$ is given by the point where $u(x, t)$ reaches a maximum. We take the maximum value of $u(x, t), u_{\max }(t) \equiv u\left(x_{c}, t\right)$, as a measure of the instantaneous amplitude scale. Near blow-up we expect $u_{\max } \sim \mathrm{U} \sim \mathrm{T}^{-1 / 2}$ and $u_{t} \sim \mathrm{U} / \mathrm{T} \sim \mathrm{T}^{-3 / 2}$. Consequently if $\partial_{t} u_{\max } \sim u_{\max }^{3}$ the self-similar scaling of the amplitude is being approached as $\mathrm{U} \rightarrow \infty$; this prediction is verified in Fig. 6(left). Likewise, using the relation $\mathrm{L} \sim \mathrm{U}^{-1 / 2}$ to provide a measure of the lengthscale in terms of $u_{\max }$ we can plot convergence of the rescaled $u(x, t)$ profiles to a normalized self-similar solution $\bar{U}(\eta)$, see Fig. 6(right).

The change of variables (4.4) transforms (4.3) into the similarity PDE,

$$
U_{s}=-\frac{1}{2} U-\frac{1}{4} \eta U_{\eta}-\left(U^{2}\right)_{\eta \eta}-U_{\eta \eta \eta \eta}
$$

Steady states of (4.5) correspond to self-similar blow-up solutions of (4.3) whose stability will be determined via linear stability analysis in similarity variables. 

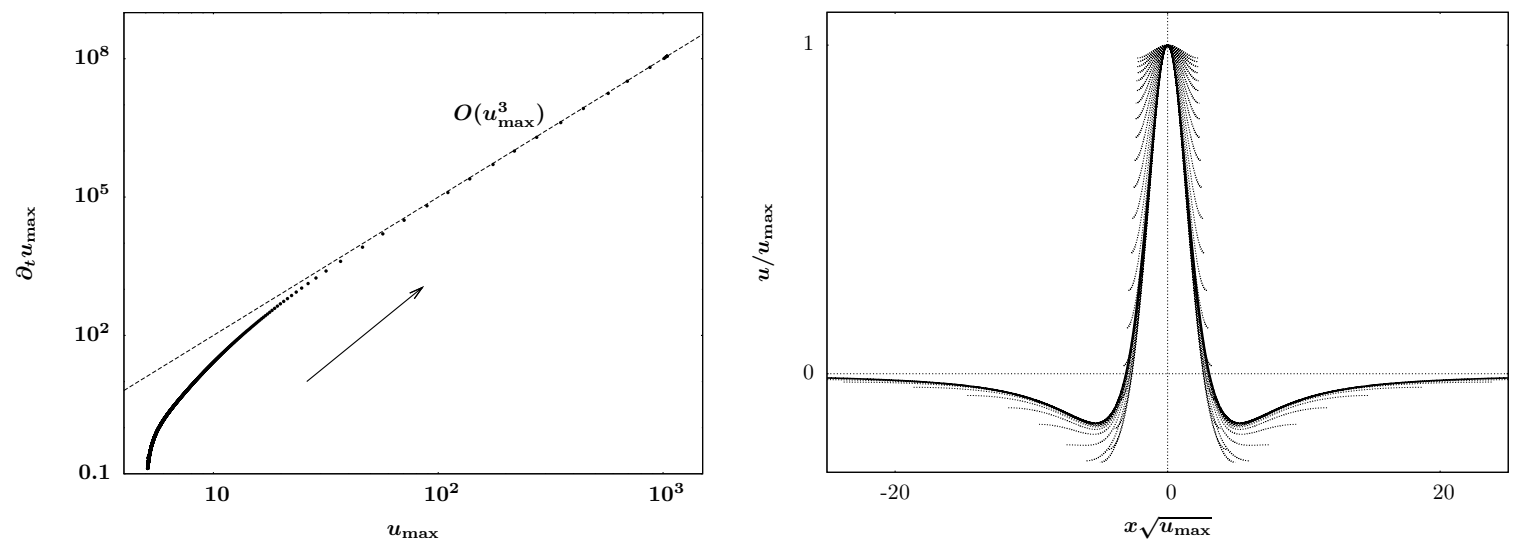

Figure 6. Convergence of the simulation from Fig. 5 to finite-time self-similar blow-up: (left) convergence of the simulation (dots) to the amplitude scaling relation $\mathrm{U}=O\left(\mathrm{~T}^{-1 / 2}\right)$ and (right) rescaled spatial profiles (dotted curves) converging to a normalized similarity solution $\bar{U}_{1}(\eta)$ (solid curve, envelope).

The choice of boundary conditions for (4.5) needs some discussion. For localized blow-up, at any fixed $x$ away from $x_{c}$ as $\tau \rightarrow 0$, the solution can be expected to remain bounded, $u(x, t)=O(1)$. This suggests that in the rescaled variables $U \sim O\left(\tau^{1 / 2}\right) \rightarrow 0$ as $|\eta| \rightarrow \infty$. This boundary condition may be refined. While the blow-up solution diverges rapidly in a neighborhood of size $\tau^{1 / 4}$ of $x_{c}$, away from $x_{c}$ the solution will be slowly varying in time, $u_{t}=O(1)$. Written in terms of $U$, this yields a Robin boundary condition,

$$
U_{s}+\frac{1}{2} U+\frac{1}{4} \eta U_{\eta}=O\left(\tau^{3 / 2}\right) \rightarrow 0, \quad|\eta| \rightarrow \infty .
$$

More explicitly we expect that this boundary condition holds in the matching region $O(1) \gg$ $\left|x-x_{c}\right| \gg O\left(\tau^{1 / 4}\right)$.

Steady solutions in the similarity variables, $\bar{U}(\eta)$, corresponding to self-similar blow-up profiles satisfy the nonlinear ODE boundary value problem

$$
\begin{gathered}
0=-\frac{1}{2} \bar{U}-\frac{1}{4} \eta \bar{U}_{\eta}-\left(\bar{U}^{2}\right)_{\eta \eta}-\bar{U}_{\eta \eta \eta \eta}, \\
\frac{1}{2} \bar{U}+\frac{1}{4} \eta \bar{U}_{\eta}=0 \quad|\eta| \rightarrow \infty
\end{gathered}
$$

The boundary condition (4.7b) implies that in order to match to outer solutions, self-similar solutions must have the far-field structure $\bar{U}(\eta) \sim c_{0} / \eta^{2}$ for some constant $c_{0}$. For $|\eta| \rightarrow \infty$, the algebraic behavior of solutions of $(4.7 \mathrm{a})$ can be obtained as a series

$$
\bar{U}(\eta) \sim \sum_{j=0}^{\infty} \frac{c_{j}}{\eta^{2+4 j}},
$$

where the coefficients $c_{j}$ for $j=1,2,3, \cdots$ are polynomials in $c_{0}$. This might suggest the existence of a one-parameter continuous family of solutions, but numerically we find only symmetric solutions for isolated exceptional values of $c_{0}$. To understand why these 
values are isolated, consider linearizing the similarity equation (4.7a) around a particular solution for $\bar{U}(\eta)$. Applying WKB analysis for $\eta \rightarrow \pm \infty$ shows that for a given $c_{0}$, there are two exponentially growing modes, an exponentially decaying mode and an algebraic mode asymptotic to $1 / \eta^{2}$ which is associated with changes in $c_{0}$. Suppressing the two exponentially growing modes at each of $\eta \rightarrow \pm \infty$ yields four boundary conditions on this fourth-order linear homogeneous problem, which generically will have only the trivial solution, suggesting that the values of $c_{0}$ are isolated. ${ }^{2}$ In addition, equation (4.7a) has a conserved integral which can be used to argue that there are only symmetric similarity solutions [10], as is seen in Fig. 7.

The first few of a discrete, countably infinite family of self-similar solutions, $\bar{U}_{\ell}(\eta)$, for $\ell=1,2,3, \cdots$, were computed using an under-relaxed Newton-Raphson method $[1,11,73]$ and are shown in Figure 7. These solutions can be indexed by the number of maxima. Bernoff \& Bertozzi [10] showed that these solutions must have zero mass, $\int \bar{U} d \eta=0$, to satisfy conservation of mass for $u(x, t)$. Evans et al [35] give a matched asymptotic construction of the self-similar solutions for a more general version of (4.3) in the limit of a large number of extrema. Their computational approach is comparable to $[73,75,76]$ and is described in terms of a one-parameter shooting search as a function of the far-field parameter $c_{0}$. While these solutions are very reminiscent of the families of discrete localized multi-bump solutions of the Swift-Hohenberg equation, it is not clear how to embed the self-similar solutions in continuous families as in "homoclinic snaking" [26, 7]. Some results on existence and local uniqueness for multi-bump blow-up solutions have been established using geometric dynamical systems analysis [62].

The first of these solutions, $\bar{U}_{1}(\eta)$, is the profile that was observed in Fig. 6(right) from the numerical solution of an initial value problem, suggesting that it is a stable attractor describing finite-time blow-up dynamics [10].

\subsection{Linear StabiLity}

Observing numerically that the self-similar solution $\bar{U}_{1}(\eta)$ appears to be an attractor, the next step is to study its stability using linear stability analysis.

To study the stability we linearize the similarity PDE (4.5) about the steady solution, $U(\eta, s)=\bar{U}(\eta)+\epsilon \tilde{U}(\eta, s)$, yielding the problem

$$
\tilde{U}_{s}=\mathcal{L} \tilde{U}, \quad \mathcal{L} \tilde{U} \equiv-\frac{1}{2} \tilde{U}-\frac{1}{4} \eta \tilde{U}_{\eta}-2(\bar{U} \tilde{U})_{\eta \eta}-\tilde{U}_{\eta \eta \eta \eta}
$$

subject to boundary conditions (4.6) applied to $\tilde{U}$. Separating variables as $\tilde{U}(\eta, s)=\phi(\eta) e^{\lambda s}$ yields the eigenvalue problem,

$$
\lambda \phi=\mathcal{L} \phi,
$$

subject to the boundary conditions

$$
-\lambda \phi=\frac{1}{2} \phi+\frac{1}{4} \eta \phi_{\eta} \quad|\eta| \rightarrow \infty .
$$

\footnotetext{
2 Similar nonlinear problems which also yield a countable set of discrete solutions have been studied using beyond-all-orders asymptotics in [28].
} 

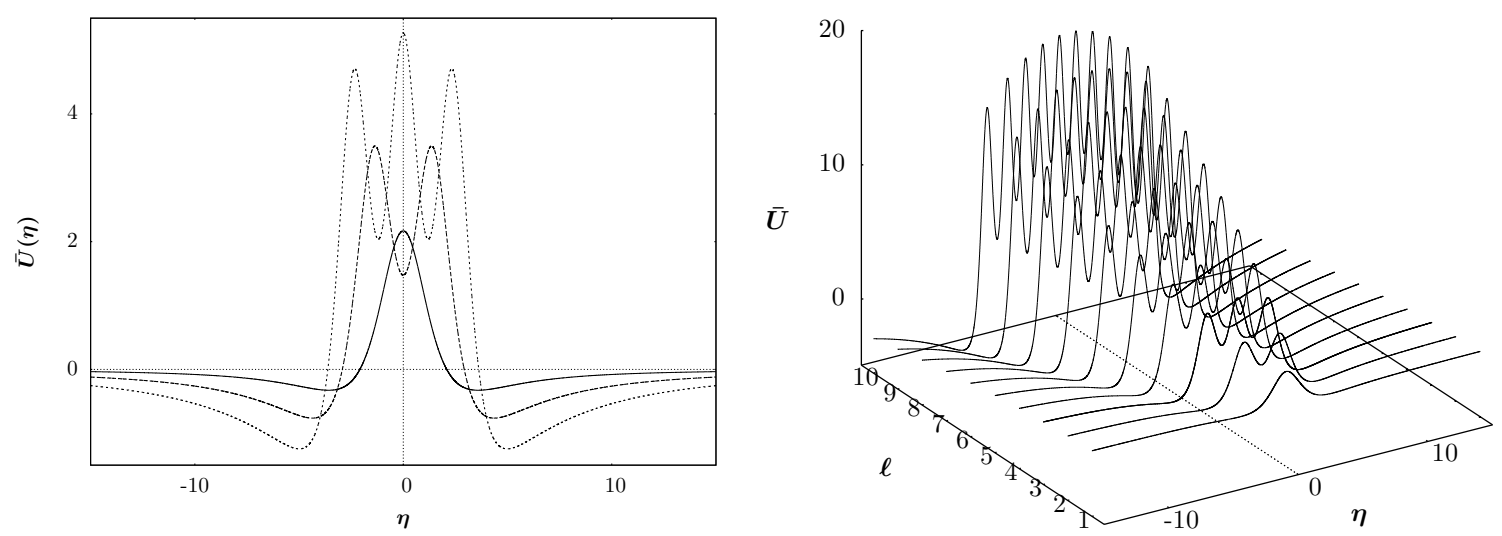

Figure \%. Multiple similarity solutions to the Sivashinsky equation: (left) the first three self-similar solutions and (right) a different view showing the first ten numerically computed self-similar solutions $\bar{U}_{\ell}(\eta)$, $\ell=1,2, \cdots, 10$.

WKB analysis of the far-field [10,35] shows that suppressing exponentially growing modes yields four boundary conditions (two each at $\eta \rightarrow \pm \infty$ ) and produces a countable discrete spectrum. Numerical calculation of the eigenvalues for the first few self-similar solutions are shown in Fig. 8; the eigenvalues are indeed discrete and all appear to be purely real.

The eigenfunctions have the far-field form $\phi=O\left(|\eta|^{-(2+4 \lambda)}\right)$ for $|\eta| \rightarrow \infty$. Note that the stable eigenmodes with $\lambda<-\frac{1}{2}$ are growing spatially for large $|\eta|$ while decaying temporally like $e^{\lambda s}$ and describe convergence in the far-field of $\eta$ of general initial conditions to the selfsimilar solutions, $\bar{U}(\eta)$. In the original variables, these modes decay like $\tau^{-\lambda-1 / 2}$ at the blow-up point.

Fig. 8 shows that there are no modes with $-\frac{1}{2} \leq \lambda \leq 0$. Modes with positive eigenvalues have eigenfunctions that decay in space more rapidly than $O\left(\eta^{-2}\right)$, so they involve corrections to the form of the solution close to the blow-up point $x_{c}$. To properly interpret the positive part of the spectrum we need to examine the role of the continuous symmetries in similarity variables.

Like the semi-linear heat equation (3.2), equation (4.3) has continuous symmetries with respect to translation in time and translation in space. As in $\S 3.5$, we consider how these symmetries act on solutions of (4.5) expressed in similarity variables.

Invariance with respect to translation in time allows us to find a one-parameter family of solutions with different blow-up times. If we set $t_{c}=t_{c}^{\prime}+\beta$, one solution of (4.3) is transformed to another, and in terms of similarity variables, when applied to a self-similar solution $\bar{U}(\eta)$, takes the form

$$
u(x, t)=\left(\tau^{\prime}\right)^{-1 / 2}\left[\left(1+\beta e^{s^{\prime}}\right)^{-1 / 2} \bar{U}\left(\eta^{\prime}\left(1+\beta e^{s^{\prime}}\right)^{-1 / 4}\right)\right]=\left(\tau^{\prime}\right)^{-1 / 2} \mathcal{U}\left(\eta^{\prime}, s^{\prime}\right) .
$$

where $\tau^{\prime}=t_{c}^{\prime}-t, \eta^{\prime}=\left(x-x_{c}\right) /\left(\tau^{\prime}\right)^{1 / 4}$, and $s=-\ln \tau^{\prime}$. Note that $\mathcal{U}\left(\eta^{\prime}, s^{\prime}\right)$ describes a one-parameter family of solutions with blow-up times shifted by $\beta$. Expanding $\mathcal{U}$ about our 


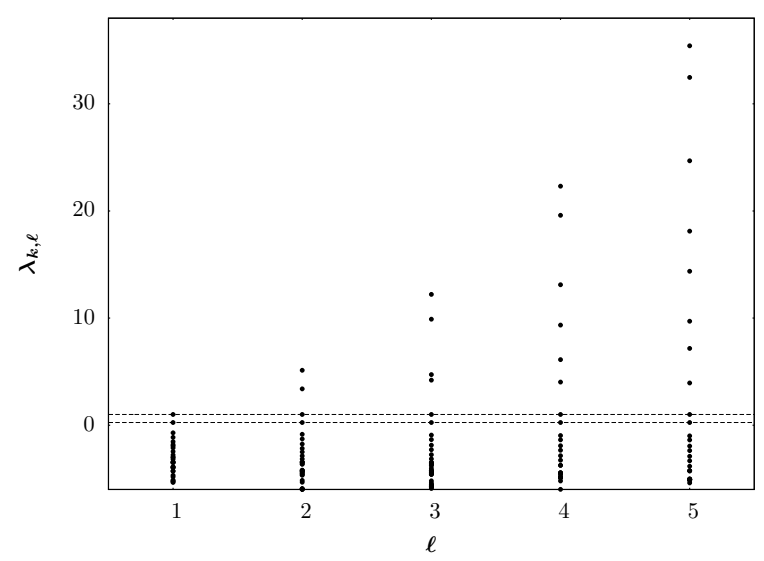

Figure 8. Positive eigenvalues for the self-similar solutions of the Sivashinsky equation. The eigenvalues at $\lambda=1 / 4,1$ are symmetry modes associated with the space and time translation symmetries (and are indicated with lines) as opposed to any true instability. The $\ell^{\text {th }}$ self-similar solution has $2 \ell$ positive eigenvalues.

original solution at $\beta=0$ yields

$$
\mathcal{U}\left(\eta^{\prime}, s^{\prime}\right) \sim \bar{U}-\beta\left(\frac{1}{2} \bar{U}+\frac{1}{4} \eta^{\prime} \frac{d \bar{U}}{d \eta}\right) e^{s^{\prime}}+O\left(\beta^{2}\right)
$$

The $O(\beta)$ term in the expansion must satisfy the linear stability problem (4.9), and we can identify the eigenmode connected with the time-translation symmetry,

$$
\phi(\eta)=\frac{1}{2} \bar{U}+\frac{1}{4} \eta \frac{d \bar{U}}{d \eta}, \quad \lambda=1 .
$$

Note that the one-parameter family of solutions is diverging exponentially in the $s$ variable (and algebraically in $\tau=t_{c}-t$ ); as such although the eigenvalue $\lambda=1$ is positive, it is a symmetry mode associated with the exponential stretching on this one-parameter manifold of blow-up solutions, not an instability. Choosing the blow-up time as the optimal-asymptotic value will remove the projection onto this mode.

Similarly, for shifts of the blow-up position, $x_{c}=x_{c}^{\prime}+\gamma$, we obtain

$$
u(x, t)=\tau^{-1 / 2} \bar{U}\left(\eta^{\prime}-\gamma e^{s / 4}\right)=\tau^{-1 / 2} \mathcal{U}\left(\eta^{\prime}, s\right)
$$

Expanding $\mathcal{U}$ for $\gamma \rightarrow 0$ yields

$$
\mathcal{U}\left(\eta^{\prime}, s\right) \sim \bar{U}-\gamma \frac{d \bar{U}}{d \eta} e^{s / 4}+O\left(\gamma^{2}\right),
$$

hence the eigenmode corresponding to spatial translation of $x_{c}$ is

$$
\phi(\eta)=\frac{d \bar{U}}{d \eta}, \quad \lambda=\frac{1}{4} .
$$

This positive eigenvalue is the symmetry mode associated with the exponential separation (in $s$ ) on the one-parameter manifold of solutions with different blow-up positions. 
Re-examining the positive spectrum of the self-similar solutions in Fig. 8 apart from the two symmetry modes we have identified, we see that the first solution is the only stable similarity solution. contains only the two symmetry modes. Each of the higher order selfsimilar solutions, $\bar{U}_{\ell}(\eta)$ for $\ell=2,3, \cdots$, has $2 \ell-2$ other positive eigenvalues, associated with true instabilities which generically lead back to the global attractor, $\bar{U}_{1}(\eta)$ [73]. The rate of convergence to $\bar{U}_{1}(\eta)$ is governed by the largest negative eigenvalue, $\lambda_{*}$. ${ }^{3}$ Since $\lambda_{*}<-\frac{1}{2}$, these contributions are of order $\tau^{-\lambda_{*}-1 / 2}$ at the blow-up point and decay in amplitude as blow-up is approached. Consequently, our stability analysis, accounting for the role of symmetries in the problem, explains why of all the similarity solutions that exist only the first one is observed in numerical simulations of the PDE.

\section{Conclusions}

In this paper we have discussed a methodology for identifying self-similar solutions and determining their stability. For each example we apply the same systematic approach; first, we use dimensional analysis to identify appropriate scalings for similarity solutions. In similarity variables, steady states of the transformed governing equation (similarity profiles) correspond to self-similar solutions in the primitive variables. We then use dynamical systems approaches to study the linear stability of these steady solutions. Spatial and temporal rescaling symmetries, whose actions are simple in the primitive variables yield parametrized families of similarity solutions which diverge (converge) exponentially in the similarity variables for finite-time blow-up (infinite-time spreading) solutions. Linearizing the actions of these symmetries yields symmetry mode contributions to the spectrum of the linearization around the similarity profiles.

Analyzing the spectrum accounting for these symmetries allows us to determine the stability of the similarity solutions. For the ODE example we demonstrate that finite-time blow-up is the generic behavior both forward and backward in time. For the semi-linear heat equation with absorption, we show that the linear spectrum can be used to identify the bifurcation between diffusion-dominated and absorption-dominated spreading and review how to perform a weakly-nonlinear bifurcation analysis at the transition between the two behaviors. For the Sivashinsky equation, linear stability identifies the unique stable similarity profile of a countably infinite set of solutions.

In conclusion, we argue that linearization methods, combined with careful analysis of the associated symmetries, provide a powerful tool for analyzing the stability of similarity solutions.

\footnotetext{
${ }^{3}$ For an example see the computations in [11], which verify that the convergence rate for perturbations to finite-time self-similar solutions match the prediction given by the largest eigenvalue from linear stability analysis.
} 


\section{Acknowledgements}

The authors wish to thank Andrea Bertozzi; as our collaborator she has contributed greatly to our understanding of self-similar phenomena. We also thank Michael Ward and George Bluman for organizing an illuminating conference on many facets of self-similarity, symmetry and scaling behavior in differential equations.

\section{References}

1. Ascher, U. M., R. M. M. Mattheij, and R. D. Russell: 1995, Numerical solution of boundary value problems for ordinary differential equations. Society for Industrial and Applied Mathematics (SIAM), Philadelphia, PA.

2. Bandle, C. and H. Brunner: 1998, 'Blowup in diffusion equations: a survey'. J. Comput. Appl. Math. 97(1-2), 3-22.

3. Barenblatt, G. I.: 1996, Scaling, self-similarity, and intermediate asymptotics, Vol. 14 of Cambridge Texts in Applied Mathematics. Cambridge: Cambridge University Press.

4. Barenblatt, G. I.: 2003, Scaling, Cambridge Texts in Applied Mathematics. Cambridge: Cambridge University Press.

5. Bebernes, J. and S. Bricher: 1992, 'Final time blowup profiles for semilinear parabolic equations via center manifold theory'. SIAM J. Math. Anal. 23(4), 852-869.

6. Bebernes, J. and D. Eberly: 1989, Mathematical problems from combustion theory, Vol. 83 of Applied Mathematical Sciences. New York: Springer-Verlag.

7. Beck, M., J. Knobloch, D. Lloyd, B. Sandstede, and T. Wagenknecht: 2008, 'Snakes, ladders, and isolas of localized patterns'. preprint.

8. Berger, M. and R. V. Kohn: 1988, 'A rescaling algorithm for the numerical calculation of blowing-up solutions'. Comm. Pur. Appl. Math 41, 841-863.

9. Bernis, F. and L. A. Peletier: 1996, 'Two problems from draining flows involving third-order ordinary differential equations'. SIAM J. Math. Anal. 27(2), 515-527.

10. Bernoff, A. J. and A. L. Bertozzi: 1995, 'Singularities in a Modified Kuramoto-Sivashinsky equation describing interface motion for phase transition'. Physica D 85, 375-404.

11. Bernoff, A. J., A. L. Bertozzi, and T. P. Witelski: 1998, 'Axisymmetric surface diffusion: dynamics and stability of self-similar pinchoff'. J. Statist. Phys. 93(3-4), 725-776.

12. Bernoff, A. J. and J. F. Lingevitch: 1994, 'Rapid relaxation of an axisymmetric vortex'. Physics of Fluids 6(11), 3717-3723.

13. Bertozzi, A. L. and M. C. Pugh: 1998, 'Long-wave instabilities and saturation in thin film equations'. Comm. Pure Appl. Math. 51(6), 625-661.

14. Bertozzi, A. L. and M. C. Pugh: 2000, 'Finite-time blow-up of solutions of some long-wave unstable thin film equations'. Indiana Univ. Math. J. 49(4), 1323-1366.

15. Bluman, G. W. and J. D. Cole: 1974, Similarity methods for differential equations. Springer-Verlag, New York-Heidelberg. Applied Mathematical Sciences, Vol. 13.

16. Bluman, G. W. and S. Kumei: 1989, Symmetries and differential equations. Springer-Verlag, New York-Berlin.

17. Boatto, S., L. P. Kadanoff, and P. Olla: 1993, 'Traveling-wave solutions to thin-film equations'. Phys. Rev. E 48(6), 4423-4431.

18. Brenner, M. P., J. R. Lister, and H. A. Stone: 1996, 'Pinching threads, singularities and the number $0.0304 \cdots$.. Phys. Fluids 8(11), 2827-2836.

19. Brezis, H., L. A. Peletier, and D. Terman: 1986, 'A very singular solution of the heat equation with absorption'. Arch. Rational Mech. Anal. 95(3), 185-209. 
20. Bricmont, J. and A. Kupiainen: 1996, 'Stable non-Gaussian diffusive profiles'. Nonlinear Anal. 26(3), 583-593.

21. Bricmont, J., A. Kupiainen, and G. Lin: 1994, 'Renormalization group and asymptotics of solutions of nonlinear parabolic equations'. Comm. Pure Appl. Math. 47(6), 893-922.

22. Budd, C. J., V. A. Galaktionov, and J. F. Williams: 2004, 'Self-similar blow-up in higher-order semilinear parabolic equations'. SIAM J. Appl. Math. 64(5), 1775-1809 (electronic).

23. Budd, C. J. and M. D. Piggott: 2001, 'The geometric integration of scale-invariant ordinary and partial differential equations'. J. Comp. and Appl. Math. 128, 399-422.

24. Budd, C. J. and M. D. Piggott: 2003, 'Geometric integration and applications'. In: Handbook of Numerical Analysis, Vol XI. Amsterdam: Elsevier-North Holland, pp. 35-139.

25. Budd, C. J., V. Rottschäfer, and J. F. Williams: 2005, 'Multibump, blow-up, self-similar solutions of the complex Ginzburg-Landau equation'. SIAM J. Appl. Dyn. Syst. 4(3), 649-678 (electronic).

26. Burke, J. and E. Knobloch: 2007, 'Homoclinic snaking:structure and stability'. Chaos 17(3), 037102.

27. Carr, J.: 1981, Applications of centre manifold theory. Springer-Verlag, New York-Berlin.

28. Chapman, S. J., J. R. King, and K. L. Adams: 1998, 'Exponential asymptotics and Stokes lines in nonlinear ordinary differential equations'. R. Soc. Lond. Proc. Ser. A Math. Phys. Eng. Sci. 454(1978), 2733-2755.

29. Childress, S. and E. A. Spiegel: 2004, 'Pattern formation in a suspension of swimming microorganisms: nonlinear aspects'. In: A celebration of mathematical modeling. Dordrecht: Kluwer Acad. Publ., pp. $33-52$.

30. Dresner, L.: 1999, Applications of Lie's theory of ordinary and partial differential equations. Bristol: Institute of Physics Publishing.

31. Eggers, J. and M. A. Fontelos: 2009, 'The role of self-similarity in singularities of partial differential equations'. Nonlinearity 22(1), R1-R44.

32. Eggers, J. and E. Villermaux: 2008, 'Physics of liquid jets'. Rep. Prog. Phys. 71(036601), 1-79.

33. Evans, J. D., V. A. Galaktionov, and J. R. King: 2007a, 'Source-type solutions of the fourth-order unstable thin film equation'. European J. Appl. Math. 18(3), 273-321.

34. Evans, J. D., V. A. Galaktionov, and J. R. King: 2007b, 'Unstable sixth-order thin film equation. I. Blow-up similarity solutions'. Nonlinearity 20(8), 1799-1841.

35. Evans, J. D., V. A. Galaktionov, and J. F. Williams: 2006, 'Blow-up and global asymptotics of the limit unstable Cahn-Hilliard equation'. SIAM J. Math. Anal. 38(1), 64-102.

36. Filippas, S. and R. V. Kohn: 1992, 'Refined asymptotics for the blowup of $u_{t}-\Delta u=u^{p}$. Comm. Pure Appl. Math. 45(7), 821-869.

37. Flores, G., G. Mercado, J. A. Pelesko, and N. Smyth: 2007, 'Analysis of the Dynamics and Touchdown in a Model of Electrostatic MEMS'. SIAM Journal on Applied Mathematics 67(2), 434-446.

38. Galaktionov, V. A., S. P. Kurdyumov, and A. A. Samarskiľ: 1985, "Asymptotic "eigenfunctions" of the Cauchy problem for a nonlinear parabolic equation'. Mat. Sb. (N.S.) 126(168)(4), 435-472, 592.

39. Galaktionov, V. A. and J. L. Vázquez: 2002, 'The problem of blow-up in nonlinear parabolic equations'. Discrete Contin. Dyn. Syst. 8(2), 399-433. Current developments in partial differential equations (Temuco, 1999).

40. Galaktionov, V. A. and J. L. Vázquez: 2004, A stability technique for evolution partial differential equations: A dynamical systems approach, Progress in Nonlinear Differential Equations and their Applications, 56. Boston, MA: Birkhäuser Boston Inc.

41. Galaktionov, V. A. and J. F. Williams: 2004, 'On very singular similarity solutions of a higher-order semilinear parabolic equation'. Nonlinearity 17(3), 1075-1099.

42. Gallay, T. and C. E. Wayne: 2002, 'Invariant manifolds and the long-time asymptotics of the NavierStokes and vorticity equations on $\mathbf{R}^{2}$. Arch. Ration. Mech. Anal. 163(3), 209-258.

43. Gandarias, M. L. and N. H. Ibragimov: 2008, 'Equivalence group of a fourth-order evolution equations unifyin various non-linear models'. Comm. Nonlinear Sci. and Num. Sim. 13, 259-268.

44. Giga, Y. and R. V. Kohn: 1985, 'Asymptotically self-similar blow-up of semilinear heat equations'. Comm. Pur. Appl. Math. 38, 297-319. 
45. Giga, Y. and R. V. Kohn: 1987, 'Characterizing Blowup Using Similarity Variables'. Indiana University Mathematics Journal 36(1), 1-40.

46. Guckenheimer, J. and P. Holmes: 1990, Nonlinear oscillations, dynamical systems, and bifurcations of vector fields, Vol. 42 of Applied Mathematical Sciences. New York: Springer-Verlag.

47. Guo, Y. J., Z. G. Pan, and M. J. Ward: 2005, 'Touchdown and pull-in voltage behavior of a MEMS device with varying dielectric properties'. SIAM Journal on Applied Mathematics 66(1), 309-338.

48. Hocherman, T. and P. Rosenau: 1993, 'On KS-type equations the evolution and rupture of a liquid interface'. Physica D 67.

49. Kadanoff, L. P.: 1997, 'Singularities and Blowups'. Physics Today 50(9), 11-13.

50. Kamin, S. and L. A. Peletier: 1985, 'Singular solutions of the heat equation with absorption'. Proc. Amer. Math. Soc. 95(2), 205-210.

51. Kleinstein, G. and L. Ting: 1971, 'Optimum one-term solutions for heat conduction problems'. $Z$. Angew. Math. Mech. 51, 1-16.

52. Kloosterziel, R. C.: 1990, 'On the large-time asymptotics of the diffusion equation on infinite domains'. J. Engrg. Math. 24(3), 213-236.

53. Levine, H. A.: 1973, 'Some nonexistence and instability theorems for solutions of formally parabolic equations of the form $P u_{t}=-A u+\mathcal{F}(u)$ '. Arch. Rational Mech. Anal. 51, 371-386.

54. Levine, H. A.: 1989, 'Quenching, nonquenching, and beyond quenching for solution of some parabolic equations'. Annali di mathematica pura ed applicata 155(4), 243-260.

55. Levine, H. A.: 1990, 'The role of critical exponents in blowup theorems'. SIAM Rev. 32(2), 262-288.

56. Merle, F. and H. Zaag: 2002, 'O.D.E. type behavior of blow-up solutions of nonlinear heat equations'. Discrete Contin. Dyn. Syst. 8(2), 435-450. Current developments in partial differential equations (Temuco, 1999).

57. Novick-Cohen, A.: 1990, 'On Cahn-Hilliard type equations'. Nonlinear Anal. 15(9), 797-814.

58. Novick-Cohen, A.: 1992, 'Blow up and growth in the directional solidification of dilute binary alloys'. Appl. Anal. 47(4), 241-257.

59. Olver, P. J.: 1993, Applications of Lie groups to differential equations. Springer-Verlag, New York.

60. Oron, A., S. H. Davis, and S. G. Bankoff: 1997, 'Long-scale evolution of thin liquid films'. Rev. Mod. Phys. 69(3), 931-980.

61. Palais, B.: 1988, 'Blowup for Nonlinear Equations Using a Comparison Principle in Fourier Space'. Commun. Pure Appl. Math. XLI, 165-196.

62. Rottschäfer, V. and T. J. Kaper: 2003, 'Geometric theory for multi-bump, self-similar, blowup solutions of the cubic nonlinear Schrödinger equation'. Nonlinearity 16(3), 929-961.

63. Samarskii, A. A., V. A. Galaktionov, S. P. Kurdyumov, and A. P. Mikhailov: 1995, Blow-up in quasilinear parabolic equations. Walter de Gruyter \& Co., Berlin.

64. Sarocka, D. C., A. J. Bernoff, and L. F. Rossi: 1999, 'Large-amplitude solutions to the Sivashinsky and Riley-Davis equations for directional solidification'. Phys. D 127(3-4), 146-176.

65. Sivashinsky, G. I.: 1983, 'On cellular instability in the solidification of a dilute binary alloy'. Phys. D 8(1-2), 243-248.

66. Stevens, A. and H. G. Othmer: 1997, 'Aggregation, Blowup, and Collapse'. SIAM Journal on Applied Mathematics 57(4), 1044-1081.

67. Straughan, B.: 1998, Explosive instabilities in mechanics. Berlin: Springer-Verlag.

68. Tanner, L. H.: 1979, 'The spreading of silicone oil drops on horizontal surfaces'. Journal of Physics D: Applied Physics 12(9), 1473-1484.

69. Vaynblat, D., J. R. Lister, and T. P. Witelski: 2001a, 'Rupture of Thin Viscous Films by van der Waals Forces I: Evolution and Self-similarity'. Physics of Fluids.

70. Vaynblat, D., J. R. Lister, and T. P. Witelski: 2001b, 'Symmetry and self-similarity in rupture and pinchoff: A geometric bifurcation'. European Journal of Applied Mathematics.

71. Velazquez, J. J., V. A. Galaktionov, and M. A. Herrero: 1991, 'The space structure near a blow-up point for semilinear heat equations: A formal approach'. Comput. Maths. Math. Phys. 31(3), 46-55. 
72. Wayne, C. E.: 1997, 'Invariant manifolds for parabolic partial differential equations on unbounded domains'. Arch. Rational Mech. Anal. 138(3), 279-306.

73. Witelski, T. P.: 2002, 'Computing finite-time singularities in interfacial flows'. In: Modern methods in scientific computing and applications (Montréal, QC, 2001), Vol. 75 of NATO Sci. Ser. II Math. Phys. Chem. Dordrecht: Kluwer Acad. Publ., pp. 451-487.

74. Witelski, T. P. and A. J. Bernoff: 1998, 'Self-similar asymptotics for linear and nonlinear diffusion equations'. Studies in Applied Mathematics 100(2), 153-193.

75. Witelski, T. P. and A. J. Bernoff: 1999, 'Stability of self-similar solutions for van der Waals driven thin film rupture'. Phys. Fluids 11(9), 2443-2445.

76. Witelski, T. P. and A. J. Bernoff: 2000, 'Dynamics of three-dimensional thin film rupture'. Phys. D 147(1-2), 155-176.

77. Witelski, T. P., A. J. Bernoff, and A. L. Bertozzi: 2004, 'Blowup and dissipation in a critical-case unstable thin film equation'. European J. Appl. Math. 15(2), 223-256.

78. Zel'dovich, Y. B. and Raizer, Yu. P.: 2002, Physics of shock waves and high-temperature hydrodynamic phenomena. New York: Dover.

79. Zhang, W. W. and J. R. Lister: 1999, 'Similarity solutions for van der Waals rupture of a thin film on a solid substrate'. Phys. Fluids 11(9), 2454-2462. 
bw2008.tex; 23/09/2011; 13:06; p.28 